\title{
Huge magnetically coupled orbital moments of Co porphyrin molecules and their control by $\mathrm{CO}$ adsorption
}

\author{
Christian F. Hermanns, ${ }^{1}$ Matthias Bernien, ${ }^{1}$ Alex Krüger, ${ }^{1}$ Waldemar Walter, ${ }^{1}$ Yin-Ming Chang (張銀銘), ${ }^{1}$ \\ Eugen Weschke, ${ }^{2}$ and Wolfgang Kuch ${ }^{1, *}$ \\ ${ }^{1}$ Institut für Experimentalphysik, Freie Universität Berlin, Arnimallee 14, 14195 Berlin, Germany \\ ${ }^{2}$ Helmholtz-Zentrum Berlin für Materialien und Energie, Albert-Einstein-Straße 15, 12489 Berlin, Germany
}

(Received 22 April 2013; revised manuscript received 14 August 2013; published 23 September 2013)

\begin{abstract}
Using x-ray magnetic circular dichroism, we show that Co porphyrin molecules adsorbed on graphenecovered Ni surfaces possess a huge in-plane (IP) orbital magnetic moment of the Co ions, despite their fourfold coordination, even comparable in size to the spin moment. Both are antiferromagnetically coupled to the $\mathrm{Ni}$ spins, leading to IP magnetization at zero external magnetic field. Adsorption of carbon monoxide on top of the molecules reduces the Co orbital moment by $(77 \pm 6) \%$. This is attributed to the change of the crystal field by the chemical stimulus which determines, together with the spin-orbit coupling (SOC), the orbital moment. Thermal desorption of the $\mathrm{CO}$ fully restores the initial electronic and magnetic properties.
\end{abstract}

DOI: 10.1103/PhysRevB.88.104420～PACS number(s): 75.70.-i, 33.15.Kr, 75.30.Et, 78.70.Dm

\section{INTRODUCTION}

Free transition metal (TM) atoms possess their highest possible multiplicity and the largest orbital moment $\left(m_{L}\right)$ following Hund's rules. Individual TM atoms on alkali films still display these physical properties due to the localization of the $3 d$ states. $^{1}$ However, crystal field (CF) effects as well as hybridization, a requirement for magnetic coupling, quench $m_{L}$ (Ref. 2) and can also affect the spin state. Both $m_{L}$ and the spin moment $\left(m_{S}\right)$ decrease as the atomic coordination is increased, passing from adsorbed single atoms ${ }^{3}$ to magnetically coupled TM atoms inside free ${ }^{4,5}$ and adsorbed clusters, ${ }^{3}$ monoatomic chains, ${ }^{6}$ two-dimensional films, ${ }^{7}$ and bulk, while generally $m_{S}>m_{L}$, as $m_{L}$ is more sensitive to modifications of the crystal field. ${ }^{4}$ Nevertheless, spin-orbit coupling (SOC) can recreate an anisotropic $m_{L}$ (Ref. 8) where values of $m_{L} / m_{S}<0.08$ for TM atoms have been observed in bulk. ${ }^{9}$ In planar organometallic complexes absolute values of $m_{L}$ can be as high as $0.53 \mu_{B}$ for bulk material ${ }^{10}$ and $0.20 \mu_{B}$ on metal surfaces. ${ }^{11}$ The anisotropic $m_{L}$ also determines the orientation of the magnetic moments of nanostructures at interfaces $^{12}$ akin to metallic thin films ${ }^{13}$ via the SOC.

Metalloporphyrins on ferromagnetic substrates contain fivefold coordinated TM ions, featuring substrate-induced magnetic ordering of unpaired spins caused by an exchange coupling to the substrate, ${ }^{14,15}$ while a minor orbital moment contributes to the magnetization, as it is linked via SOC to $m_{s} \cdot{ }^{14}$ Recently, it has been shown that an antiferromagnetic (AFM) coupling between Co porphyrin molecules and Ni films can be established even across graphene. ${ }^{16}$ The immobilization of such planar molecules on magnetic surfaces makes them technologically appealing, as one of the two axial coordination sites of the central metal ion can still be occupied by an additional ligand. It has already been demonstrated that the adsorption of small molecules on top can manipulate the magnetization of adsorbed metalloporphyrins. ${ }^{17-22}$ In these previous studies the magnetic properties have been modified by a change of the spin moment of the central metal ion or by a change in the magnetic coupling to a ferromagnetic substrate, while the contribution of the orbital moment was negligible.
Here, we present an angle-, field-, and temperaturedependent $\mathrm{x}$-ray absorption spectroscopy (XAS) and x-ray magnetic circular dichroism (XMCD) study of submonolayers of Co octaethylporphyrin (CoOEP) molecules and CO-CoOEP carbonyl complexes on a graphene-protected $\mathrm{Ni}$ film grown on a W(110) surface. For both systems the Co ions are antiferromagnetically coupled to the substrate magnetization. In the case of CoOEP, a substrate-induced in-plane (IP) aligned extraordinarily huge Co orbital moment of $m_{L}=(0.93 \pm$ 0.05) $\mu_{B}$ contributes to the same extent to the Co magnetization as the spin moment. The orbital moment reveals an anisotropy in size of $489 \%$. After dosing carbon monoxide (CO), the formation of CO-CoOEP carbonyl complexes is concluded from changes in the Co $L_{2,3} \mathrm{XA}$ spectra. Thereby the IP aligned Co $m_{L}$ reduces by $(77 \pm 6) \%$, compared to the pristine state, due to $\mathrm{CO}$-induced modifications of the crystal field. This demonstrates the possibility to control not only $m_{S}$, but also $m_{L}$ of exchanged-coupled magnetic moments of organometallic complexes by means of the chemical stimulus $\mathrm{CO}$.

\section{EXPERIMENT}

X-ray absorption spectrosopy (XAS) measurements were carried out at the PM-3 and UE46-PGM1 beamlines at the synchotron radiation source BESSY II in Berlin with energy resolutions of 300 and $150 \mathrm{meV}$, respectively, by means of total electron yield detection. The spectra were normalized to the total electron yield of a freshly evaporated gold grid upstream to the experiment at the PM-3 beamline, while at UE46-PGM1 the drain current from the last refocussing mirror upstream from the measuring chamber had been used. At both beamlines the spectra were further normalized to the corresponding spectra of the clean substrate, and scaled to 1 in the pre-edge energy region. Circularly polarized photons with $90 \%$ and $85 \%$ degree of polarization were used at UE46PGM1 (Fig. 1) and PM-3 (Fig. 4), respectively. At the PM-3, samples were prepared and measured in ultrahigh vacuum with a base pressure of $2.0 \times 10^{-10}$ mbar. At UE46-PGM1, the pressures of the experimental station for the sample preparation and the measurements were $1.0 \times 10^{-9}$ and 
$1.0 \times 10^{-10}$ mbar, respectively. Co(II) $2,3,7,8,12,13,17,18$ octaethylporphyrin (CoOEP) molecules were sublimated on an ordered graphene layer, prepared by cracking of propylene gas $\left(\mathrm{C}_{3} \mathrm{H}_{6}\right)$ on Ni films, which were epitaxially grown by electronbeam evaporation on a W(110) single crystal surface. ${ }^{23,24}$ More details about sample preparation, calibration of the photon energy, and coverages are published elsewhere. ${ }^{16}$ Radiation damage could be excluded from comparison of spectra taken immediately after sample preparation and at later times.

\section{RESULTS AND DISCUSSION}

First we focus on the magnetic properties of the adsorbed CoOEP molecules, and in particular on the contribution of $m_{L}$ to the Co magnetization. We present in Fig. 1 Co $L_{2,3}$ helicityaveraged XA spectra (a) and the corresponding XMCD spectra
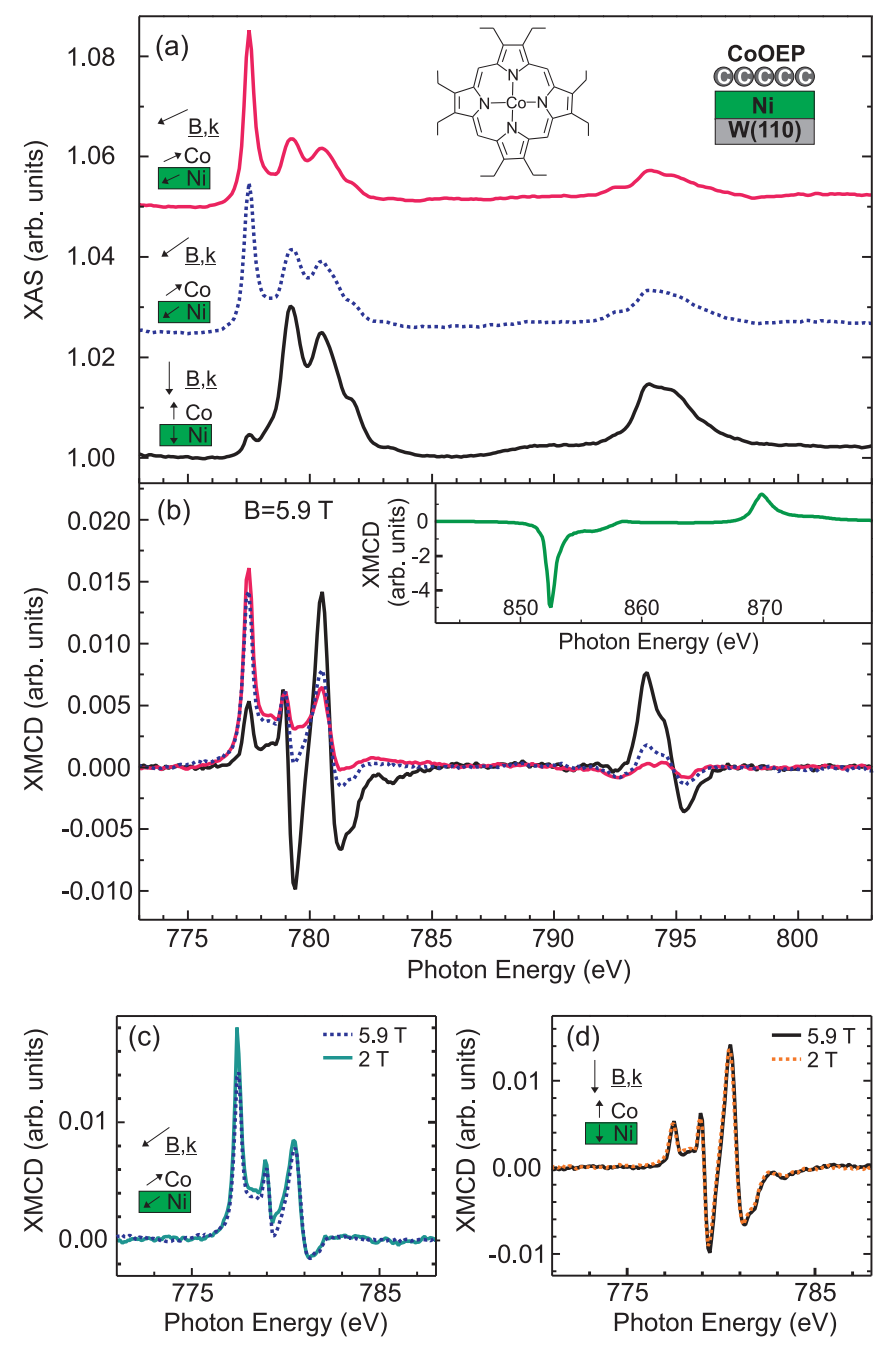

FIG. 1. (Color online) Co $L_{2,3}$ helicity-averaged XA spectra (a) and corresponding XMCD spectra (b) of 0.8 ML CoOEP on graphene/25 ML Ni/W(110) measured at $4.5 \mathrm{~K}$ in a magnetic field of $B=5.9 \mathrm{~T}$. Spectra taken at $25^{\circ}$ (pink lines) and $35^{\circ}$ (blue dashed lines) grazing and at normal (dark lines) incidence are shown. The Co XA spectra are shifted vertically for clarity. Panels (c) and (d) display Co $L_{3} X \mathrm{XMCD}$ spectra recorded at $35^{\circ}$ grazing and normal incidence at two different field strengths of $2 \mathrm{~T}$ (cyan and dashed orange lines) and 5.9 T, respectively. (b) of $0.8 \mathrm{ML}$ (monolayer) CoOEP on graphene/25 ML Ni/W(110), measured at a temperature of $T=4.5 \mathrm{~K}$ in an external magnetic field $\left(B_{\text {ext }}\right)$ of $5.9 \mathrm{~T}$, applied along the corresponding X-ray incidence direction. Spectra taken at $25^{\circ}$ (pink lines) and $35^{\circ}$ (blue dashed lines) grazing and at $90^{\circ}$ normal (black lines) incidence are shown. DFT calculations had revealed that the Co ions of the flatly adsorbed molecules are in a $d^{7}$ low-spin state, where the energetically highest $b_{1 \mathrm{~g}}$ $\left(d_{\mathrm{x}^{2}-\mathrm{y}^{2}}\right)$ orbital is empty and the $a_{1 \mathrm{~g}}\left(d_{\mathrm{z}^{2}}\right)$ orbital is half filled. ${ }^{16}$ The Co XA spectra exhibit a detailed fine structure at the $L_{3}$ edge. The narrow peak at $777.5 \mathrm{eV}$ and energetically higherlying peaks of the Co $L_{3}$ XA spectra are more pronounced in the spectra recorded at $25^{\circ}$ grazing and normal incidence, respectively, as expected for excitations into out-of-plane and in-plane orbitals. Hence, the angular dependence and the energetic position between the components building up the $L_{3}$ edge are in line with the orbital occupation as obtained from DFT calculations.

The isotropic Co $L_{3}$ XMCD spectrum, measured at the magic angle of $35^{\circ}$ grazing incidence, has a main peak at $777.8 \mathrm{eV}$ and at higher energies two local maxima at 779.3 and $780.8 \mathrm{eV}$. The former is more prominent at $25^{\circ}$ grazing incidence, the latter two at normal incidence, at which zero-crossings lead to a fine structure with a double dip shape. The isotropic Co and Ni $L_{3}$ XMCD signals, the latter shown in the inset of Fig. 1(b), have different signs, which proves an antiferromagnetic coupling between the magnetization of the substrate and the spins of the Co ions that is stronger than the Zeemann interaction. The external magnetic field forces the Ni magnetization along the $\mathrm{X}$-ray incidence direction (see Supplemental Material, Fig. S1), ${ }^{25}$ and the antiferromagnetically coupled Co moments align accordingly. The Co $L_{2,3}$ $\mathrm{XMCD}$ line shape is rather exceptional, featuring several zero crossings at normal incidence.

Figures 1(c) and 1(d) compare Co $L_{3}$ XMCD spectra, which were recorded at $35^{\circ}$ grazing incidence and normal incidence, respectively, in an external magnetic field of $2 \mathrm{~T}$ (full cyan and dashed orange lines) and 5.9 $\mathrm{T}$ at $4.5 \mathrm{~K}$. Apparently, the XMCD signals match each other in size at normal but not at grazing incidence, which shows that in the former case the Co magnetization is identically aligned at the two field strengths. At both detection angles, Ni $L_{2,3}$ XMCD spectra (see Supplemental Material, Fig. S1) ${ }^{25}$ reveal a virtually fully aligned substrate magnetization, regardless whether a magnetic field of 2 or $5.9 \mathrm{~T}$ is applied. Consequently, the counteraction of the applied magnetic field against the AFM coupling between the magnetization of the Ni films and the Co spins is big enough at 5.9 $\mathrm{T}$ to reduce the Co magnetization at grazing incidence by $(17 \pm 2) \%$. Both an in-plane easy axis, as suggested by the anisotropy of $m_{L}$ and reported for a similar system, ${ }^{26}$ as well as the different Zeeman energies would lead to the opposite behavior. Hence, our finding must be explained by an orbitally-dependent exchange interaction between the Co and Ni spins that is magnetically anisotropic. ${ }^{27}$ This has already been observed for single molecular spins on magnetic surfaces. $^{28,29}$

Referring to the line shape of the angle-dependent Co $\mathrm{XAS}$ and XMCD spectra, a comparison to results from $\mathrm{CoPc}$ bulk measurements ${ }^{30}$ lets us conclude that the SOC mixes the ground state and the first excited state of the electronic 
structure of the Co ions. This results in a nonvanishing hole density at the $e_{g}$ orbital and a more than half filled $a_{1 g}$ orbital, and thus creates an IP $m_{L}{ }^{8}$

The orbital and spin moments can be quantified by applying the XMCD sum rules ${ }^{31,32}$ to the angle-dependent Co XMCD spectra presented in Figs. 1(a) and 1(b). Thereby three holes within the Co $3 d$ orbitals are assumed, the reduction of the Co magnetization by the applied magnetic field is taken into account, and the integrated helicity-averaged Co XA spectrum, taken at $35^{\circ}$ grazing incidence, is used as isotropic intensity. For flatly adsorbed molecules and an angle of incidence $\alpha$ between the wave vector of the $\mathrm{x}$ rays and the surface plane, the experimentally measured orbital moment is composed of two angle-dependent components, assuming fourfold rotational symmetry of the molecules leading to a uniaxial anisotropy along the surface normal: ${ }^{33}$

$$
m_{L}(\alpha)=m_{L}^{z} \sin ^{2}(\alpha)+m_{L}^{x y} \cos ^{2}(\alpha),
$$

where $m_{L}^{z}$ and $m_{L}^{x y}$ are the orbital moments in the $z$ axis and the average in the $x-y$ plane, respectively. The effective spin moment contains two contributions, ${ }^{10}$

$$
m_{S}^{\text {eff }}(\alpha)=m_{S}-7 \underbrace{\left[m_{T}^{z} \sin ^{2}(\alpha)+m_{T}^{x y} \cos ^{2}(\alpha)\right]}_{m_{T}(\alpha)},
$$

where $m_{S}$ is the spin moment and the term $7 m_{T}(\alpha)$ stands for the intra-atomic spin dipole moment. The latter originates from an anisotropic spatial distribution of the spin density, where $\left(m_{T}^{z}+2 m_{T}^{x y}\right)=0$. The magnetic moments evaluated from the sum rules are presented in Fig. 2 as a function of $\alpha$, combined with fits to these results following Eqs. (1) and (2). For measurements taken at the magic angle, $m_{S}^{\text {eff }}\left(35^{\circ}\right)=-(1.00 \pm$ $0.07) \mu_{B}$ is obtained, as expected for an $S=1 / 2$ system in saturation. A value of $m_{S}^{\text {eff }}\left(25^{\circ}\right)=-(1.37 \pm 0.10) \mu_{B}$ and even an opposite sign for $m_{S}^{\mathrm{eff}}\left(90^{\circ}\right)=+(0.34 \pm 0.05) \mu_{B}$ are evaluated. This is explained by an extraordinarily large anisotropy of the intra-atomic dipolar contribution within this lowsymmetry system. The fit shown in Fig. 2 yields $m_{T}\left(90^{\circ}\right)=$ $-(0.20 \pm 0.05) \mu_{B}$, which is an unusually big value, and $m_{S}=-(1.03 \pm 0.07) \mu_{B}$. The observed sign reversal of $m_{S}^{\text {eff }}$ is consistent with an unpaired hole in the $d_{z^{2}}$ orbital. ${ }^{33}$ Also the orbital moment exhibits a pronounced angular dependence. The experimentally determined out-of-plane orbital moment $m_{L}^{z}=-(0.19 \pm 0.05) \mu_{B}$ is much smaller than the ones

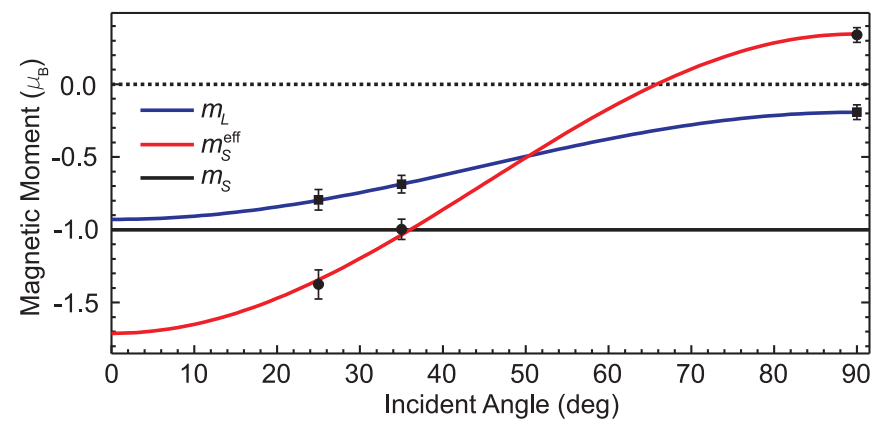

FIG. 2. (Color online) Angle dependence of the experimental expectation values of the orbital magnetic moment $m_{L}$ (squares) and the effective spin moment $m_{S}^{\text {eff }}$ (circles), shown together with fits following Eqs. (1) (blue line) and (2) (red line).

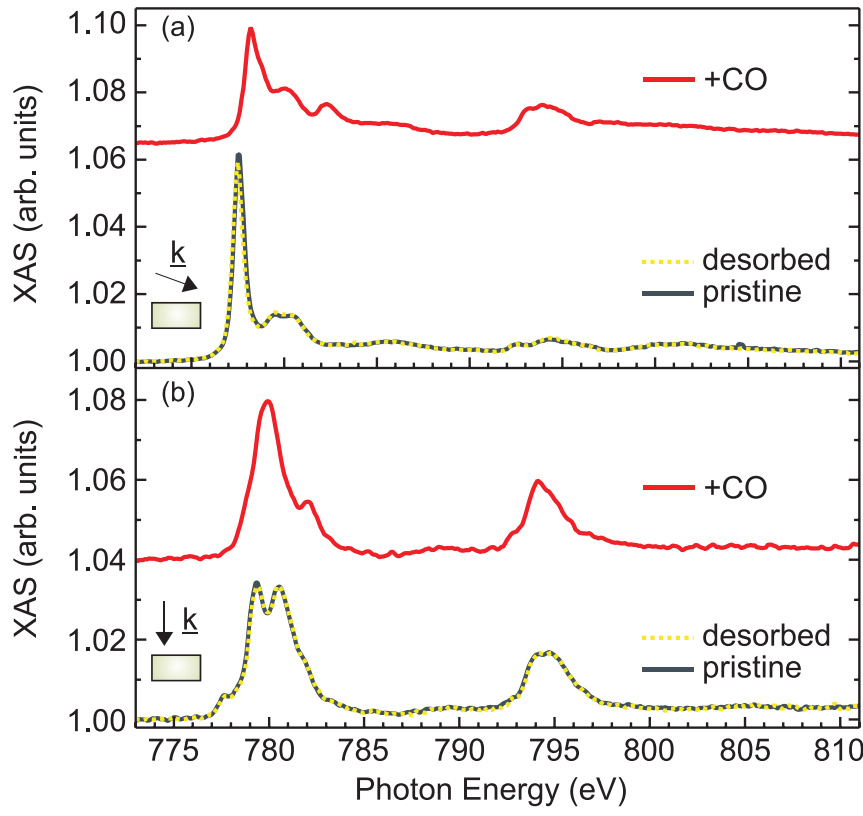

FIG. 3. (Color online) Co $L_{2,3}$ XA spectra of $0.9 \mathrm{ML}$ CoOEP on graphene/21 ML Ni/W(110) measured with $p$-linearly polarized light at an angle of $20^{\circ}$ (a) and $90^{\circ}$ (b) between the X-ray wavevector and the surface. For both subfigures, the spectra are shown for the sample in the pristine state (continuous dark line), recorded at $165 \mathrm{~K}$, after dosing with $90 \mathrm{~L}$ of $\mathrm{CO}$ (continuous red line), measured at $40 \mathrm{~K}$, and after the desorption of CO (yellow dashed lines), recorded again at $165 \mathrm{~K}$. The spectra after dosing are vertically offset for clarity.

measured at grazing incidence: $m_{L}\left(35^{\circ}\right)=-(0.69 \pm 0.06) \mu_{B}$ and $m_{L}\left(25^{\circ}\right)=-(0.80 \pm 0.07) \mu_{B}$. Remarkably, the extrapolated in-plane orbital moment $m_{L}^{x y}$ even reaches $-(0.93 \pm$ 0.10) $\mu_{B}$, which means an exceptionally large orbital moment anisotropy of $489 \%$. Hence, we observe the highest ever found ratio $m_{L}^{x y} / m_{S}=0.90$ in $3 d$ complexes and compounds, despite the hybridization of the Co orbitals, which is necessary for the magnetic coupling between the Co orbital moments and the Ni magnetization.

To manipulate the crystal field splitting, which, together with the SOC determines the size of $m_{L}$, we attach and detach $\mathrm{CO}$ to the $\mathrm{Co}$ ions as an additional axial ligand. $90 \mathrm{~L}$ of $\mathrm{CO}\left(1 \mathrm{~L}=10^{-6}\right.$ mbar s) were dosed to the sample at 40-50 K. Desorption of the CO molecules was achieved by a subsequent annealing of the sample to $165 \mathrm{~K}$. Angle-dependent XA measurements at the Co $L_{2,3}$ edges prove that the changes to the electronic structure and in particular the crystal field of the Co ions are fully reversible. Figure 3 compares Co $L_{2,3}$ X-ray absorption spectra of 0.9 ML CoOEP on graphene/21 ML Ni/W(110) taken with $p$-linearly polarized light at $165 \mathrm{~K}$ (continuous dark lines), after dosing $90 \mathrm{~L}$ of $\mathrm{CO}$, recorded at $40 \mathrm{~K}$ (continuous red lines), and after thermal desorption of $\mathrm{CO}$, measured again at $165 \mathrm{~K}$ (yellow dashed lines). XA spectra recorded at $20^{\circ}$ grazing incidence (a) and at normal incidence (b) are shown, where those of the pristine sample exhibit a qualitatively identical line shape to the helicity-averaged XA spectra taken with circular polarization presented in Fig. 1(a). Unambiguously, line-shape changes are found at both detection angles after dosing $\mathrm{CO}$, which reveal a formation of CO-CoOEP carbonyl complexes on the surface. 
A comparison of the spectral intensity at around $777.5 \mathrm{eV}$ of the Co $L_{3} \mathrm{XA}$ spectra recorded at $20^{\circ}$ grazing incidence before and after dosing with CO shows that a dosage of $90 \mathrm{~L} \mathrm{CO}$ is sufficient to coordinate more than $90 \%$ of the Co ions by a $\mathrm{CO}$ molecule, if not all. The restoration of the original line shape at the two measurement geometries after annealing the sample to $165 \mathrm{~K}$ reveals a subsequent thermal detachment of the $\mathrm{CO}$ from the Co ions, demonstrating the full reversibility of the process. By making use of the charge sum rule ${ }^{33}$ and comparing the results before and after $\mathrm{CO}$ uptake, an increase of $(6 \pm 3) \%$ for grazing incidence and a decrease of $(5 \pm 3) \%$ for normal incidence of the integrated intensity of the white lines are obtained, respectively. This shows that after dosing $\mathrm{CO}$ a small amount of electron density is withdrawn from out-of-plane orbitals and augmented at in-plane orbitals. These findings are consistent with $\mathrm{CO}$ acting as an additional axial $\sigma$-acceptor and $\pi$-donor ligand. Additionally, a shift to higher photon energies of the $\mathrm{Co}_{2} L_{3}$ XAS edge after $\mathrm{CO}$ attachment is detected for measurements at grazing incidence, while no significant influence on the energetic position is observed at normal incidence. As a result, the linear dichroism for the CO-CoOEP complexes is reduced. This is in accordance with a coordination of $\mathrm{CO}$ to the $\mathrm{Co}$ ions, which modifies the rather square planar shape of the crystal field ( $D_{4 h}$ symmetry) to a more tetragonally acentrically distorted one $\left(C_{4 v}\right.$ symmetry). ${ }^{34}$ Such a change pushes the Co $3 d$ states with out-of-plane charge distribution $e_{g}$ and $a_{1 g}$ to higher energies. The branching ratio, defined as the ratio between the integrated XA white line intensity of the $L_{3}$ edge and the sum of the $L_{3}$ and $L_{2}$ edges, decreases for the carbonyl complexes for grazing incidence from $0.80 \pm 0.03$ to $0.69 \pm 0.03$, but stays constant for normal incidence $(0.68 \pm 0.03$ vs $0.67 \pm 0.03)$. Assuming a negligible electrostatic interaction between core hole and valence electrons, the deviation of the branching ratio from the statistic value of $2 / 3$ is proportional to the angle-dependent part of the spin-orbit operator. ${ }^{35}$ The anisotropic branching ratio before $\mathrm{CO}$ uptake fits well to the above-mentioned large and anisotropic orbital moment caused by an angle-dependent spin-orbit interaction. ${ }^{36,37}$ In contrast, the rather isotropic branching ratio of the CO-CoOEP carbonyl complexes points towards a smaller influence of the spin-orbit coupling on the electronic properties of the Co ions.

In order to work out the influence of dosing with $\mathrm{CO}$ on the magnetic properties of the Co ions, Fig. 4 shows Co $L_{2,3}$ XA helicity-averaged spectra (a) of 0.9 ML CoOEP (dark green lines) and CO-CoOEP (red lines) on graphene/21 ML $\mathrm{Ni} / \mathrm{W}(110)$, recorded at $20^{\circ}$ grazing incidence, and the corresponding XMCD spectra (b). Measurements in remanence of the Ni film with its IP easy axis along the $\langle 1 \overline{1} 0\rangle$ direction, ${ }^{24}$ where the Co magnetization is aligned correspondingly, enable the recording of the IP Co XMCD at zero magnetic fields. They are taken at 125 and $40 \mathrm{~K}$, before and after dosing CO, respectively. For reasons of comparability, in the former case the Co XMCD signal is scaled up by a factor of 4 to its expected size at $40 \mathrm{~K}$, estimated from literature. ${ }^{16}$ The line shape of the XA and XMCD spectra of the pristine sample are in qualitative agreement with the respective spectra presented in Fig. 1 and are clearly different compared to the ones obtained after dosing, which shows that $\mathrm{CO}$ and $\mathrm{CoOEP}$ molecules form stable complexes. Integrating the two Co XA spectra, which

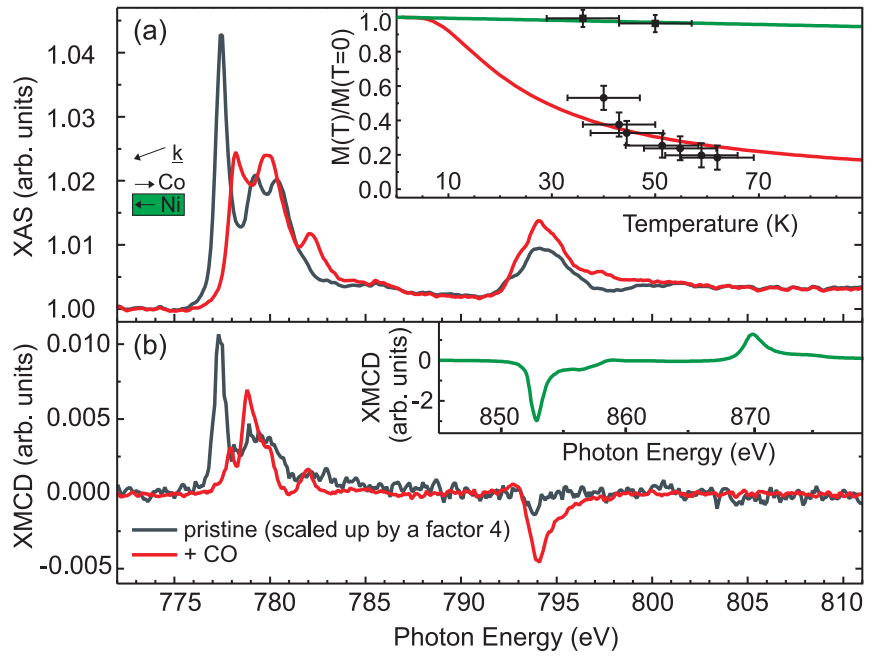

FIG. 4. (Color online) Co $L_{2,3}$ XA helicity-averaged spectra (a) of $0.9 \mathrm{ML}$ CoOEP (dark grey lines), taken at $125 \mathrm{~K}$, and CO-CoOEP (red lines), measured at $40 \mathrm{~K}$, on graphene/21 ML $\mathrm{Ni} / \mathrm{W}(110)$, and the corresponding XMCD spectra (b) recorded at $20^{\circ}$ grazing incidence and in remanence of the Ni film. Insets: (a) $M_{r}^{\mathrm{Co}}(T)$ and $M_{r}^{\mathrm{Ni}}(T)$ derived from integrated XMCD signals of CO-CoOEP (circles and squares, respectively) and presented together with the respective calculated progression (see text). (b) $\mathrm{Ni} L_{2,3} \mathrm{XMCD}$ spectra taken at $125 \mathrm{~K}$ and before dosing $\mathrm{CO}$.

are measured $15^{\circ}$ off the magic angle, reveals a CO-induced rise of $(2 \pm 3) \%$ of the white line intensity. According to this small enlargement, which is zero within the error bar, as well as the $\mathrm{CO}$ influence on the white line intensity for different measurement conditions (see Supplemental Material, Fig. S2), we conclude from the charge sum rule ${ }^{33}$ that $\mathrm{CO}$ adsorption does not alter the oxidation state of the Co ions.

The almost vanishing IP Co XMCD at the $L_{2}$ edge before dosing $\mathrm{CO}$, compared to the big intensity at the $L_{3}$ edge, reflects the huge Co in-plane orbital moment of the adsorbed CoOEP molecules. A nondisappearing Co XMCD signal for the CO-CoOEP complexes demonstrates the existence of a magnetic moment on the Co site, and simultaneously a magnetic coupling between these magnetic moments and the Ni magnetization. The most prominent changes of the $\mathrm{Co}$ XMCD spectrum upon CO uptake are a shift to higher photon energies at the $L_{3}$ edge, accompanied by a modification of the line shape, which now exhibits a main maxima at $778.9 \mathrm{eV}$ and two local maxima at 778.0 and $782.1 \mathrm{eV}$. The remarkable increase of the XMCD at the Co $L_{2}$ edge after dosing $\mathrm{CO}$ indicates a strongly reduced IP orbital moment. By utilizing the sum rules, ${ }^{31,32}$ lower limits of the absolute values of the IP orbital moment of $(0.11 \pm 0.02) \mu_{B}$ as well as of the effective spin moment of $(0.75 \pm 0.05) \mu_{B}$ are evaluated in the case of the CO-CoOEP complexes.

For a determination of the saturated magnetic moments after $\mathrm{CO}$ attachment, we study the temperature progression of the $\mathrm{Co}$ and $\mathrm{Ni} L_{3}$ edge XMCD signals of $\mathrm{CO}$ $\mathrm{CoOEP} /$ graphene/Ni and show the deduced relative $\mathrm{Co}$ and $\mathrm{Ni}$ magnetizations $\left[M_{r}^{\mathrm{Co}}(T)\right.$ and $\left.M_{r}^{\mathrm{Ni}}(T)\right]$ versus temperature in the inset of Fig. 4(a) together with their theoretical temperature progression. $M_{r}^{\mathrm{Co}}(T)$ is reproduced by a Brillouin function 
$B_{J}\left(E_{\text {ex }} / k_{B} T\right)$ with temperature $T$, Boltzmann constant $k_{B}$, $E_{\mathrm{ex}}=(1.4 \pm 0.5) \mathrm{meV}$, and $J=0.5$, including the coupling to the magnetic substrate by an effective magnetic field: ${ }^{38}$ $M_{r}^{\mathrm{Co}}(T)=M_{r}^{\mathrm{Ni}}(T) B_{J}(\alpha) . M_{r}^{\mathrm{Ni}}(T)$ is represented by a $(1-$ $\left.T / T_{C}\right)^{\beta}$ law, with a critical exponent $\beta=0.365$ and a Curie temperature of $T_{C}=630 \mathrm{~K} .{ }^{39}$ Extrapolated values of $-(0.21 \pm 0.05) \mu_{B}$ and $-(1.43 \pm 0.04) \mu_{B}$ are found for $m_{L}^{x y}$ and $m_{S}^{\text {eff }}\left(0^{\circ}\right)$, respectively. This reveals the reduction of $m_{L}^{x y}$ upon $\mathrm{CO}$ uptake by $(77 \pm 6) \%$. The value for $m_{S}^{\text {eff }}\left(0^{\circ}\right)$ is consistent with a spin state $S=1 / 2$, as expected for Co porphyrins, ${ }^{40}$ and $7 m_{T}\left(0^{\circ}\right)=(0.43 \pm 0.04) \mu_{B}$.

The explanation for the reduced Co IP orbital moment upon $\mathrm{CO}$ uptake comes from the modified Co electronic structure. In the pristine state, the $e_{g}$ and $a_{1 g}$ states are energetically almost degenerate in the CoOEP molecules adsorbed on the substrate, which allows for substantial mixing. A new state with an anisotropic orbital momentum is formed and a huge in-plane orbital moment is created. ${ }^{8}$ Within the CO-CoOEP complexes, the degeneracy is lifted: The $\mathrm{CO}$ molecules bind axially to the Co ions, such that they push up the $a_{1 g}$ state more effectively in energy than the $e_{g}$ state. ${ }^{12,34}$ Treating the recreated IP orbital moment as a second-order perturbation effect, it is inversely proportional to the energetic separation between the two states, which is bigger for the CO-CoOEP complexes. Consequently $m_{L}^{x y}$ may reduce upon $\mathrm{CO}$ uptake. An alternative explanation that we can not exclude could be a CO-induced reorganization of the Co $3 d$ orbital occupation, which might also lead to a reduced size of $m_{L}^{x y}$.

\section{CONCLUSION}

We have shown that the Co spin and orbital moments of CoOEP adsorbed on graphene-covered $\mathrm{Ni}$ films, being $-(1.03 \pm 0.07) \mu_{B}$ and $-(0.93 \pm 0.05) \mu_{B}$ for an IP aligned Co magnetization, respectively, are anisotropically exchange coupled to the $\mathrm{Ni}$ spins in an AFM fashion. Despite the hybridization of Co $3 d$ with molecular states, which enables the alignment of the Co orbital moments by the magnetic exchange interaction, ${ }^{16} m_{L}^{x y}$ and $m_{S}$ are of similar magnitude. The Co orbital moment, recreated by SOC, reveals a giant anisotropy in size of $489 \%$. The spin density is highly anisotropic, too, reflecting the out-of-plane density of unpaired holes at the Co side. By adsorbing and thermally removing $\mathrm{CO}$ to the CoOEP molecules, the electronic structure of the Co ions can be controlled in a reversible manner, while neither the oxidation state nor the substrate-induced magnetic order are changed. The attachment of $\mathrm{CO}$ modifies the $\mathrm{CF}$ of the Co ions, which, together with the SOC, determines the size of $m_{L}^{x y}$. CO adsorption reduces $m_{L}^{x y}$ by $(77 \pm 6) \%$ and thus shows that it is possible to change the magnetization within an exchange-coupled system of metalloporphyrins adsorbed on surfaces not only by varying $m_{S}$, but also by modifying $m_{L}$.

\section{ACKNOWLEDGMENTS}

T. Kachel and E. Schierle are acknowledged for their technical support. Funding by the DFG (Sfb 658) and the BMBF (05K10KE1) is gratefully acknowledged. *kuch@physik.fu-berlin.de

${ }^{1}$ P. Gambardella, S. S. Dhesi, S. Gardonio, C. Grazioli, P. Ohresser, and C. Carbone, Phys. Rev. Lett. 88, 047202 (2002).

${ }^{2}$ J. H. van Vleck, The Theory of Electric and Magnetic Susceptibilities (Clarendon, Oxford, 1932).

${ }^{3}$ P. Gambardella, S. Rusponi, M. Veronese, S. S. Dhesi, C. Grazioli, A. Dallmeyer, I. Cabria, R. Zeller, P. H. Dederichs, K. Kern, C. Carbone, and H. Brune, Science 300, 1130 (2003).

${ }^{4}$ M. Niemeyer, K. Hirsch, V. Zamudion-Bayer, A. Langenberg, M. Vogel, M. Kossick, C. Ebrecht, K. Egashira, A. Terasaki, T. Möller, B. v. Issendorff, and J. T. Lau, Phys. Rev. Lett. 108, 057201 (2012).

${ }^{5}$ S. Peredkov, M. Neeb, W. Eberhardt, J. Meyer, M. Tombers, H. Kampschulte, and G. Niedner-Schatteburg, Phys. Rev. Lett. 107, 233401 (2011).

${ }^{6}$ P. Gambardella, A. Dallmeyer, K. Maiti, M. C. Malagoli, W. Eberhardt, K. Kern, and C. Carbone, Nature (London) 416, 301 (2002).

${ }^{7}$ M. Tischer, O. Hjortstam, D. Arvanitis, J. Hunter Dunn, F. May, K. Baberschke, J. Trygg, J. M. Wills, B. Johansson, and O. Eriksson, Phys. Rev. Lett. 75, 1602 (1995).

${ }^{8}$ J. Stöhr, J. Magn. Magn. Mater. 200, 470 (1999).

${ }^{9}$ O. Eriksson, A. M. Boring, R. C. Albers, G. W. Fernando, and B. R. Cooper, Phys. Rev. B 45, 2868 (1992).

${ }^{10}$ J. Bartolomé, F. Bartolomé, L. M. García, G. Filoti, T. Gredig, C. N. Colesniuc, I. K. Schuller, and J. C. Cezar, Phys. Rev. B 81, 195405 (2010).
${ }^{11}$ S. Stepanow, A. Mugarza, G. Ceballos, P. Moras, J. C. Cezar, C. Carbone, and P. Gambardella, Phys. Rev. B 82, 014405 (2010).

${ }^{12}$ P. Gambardella, S. Stepanow, A. Dmitriev, J. Honolka, F. M. F. de Groot, M. Lingenfelder, S. S. Gupta, D. D. Sarma, P. Bencok, S. Stanescu, S. Clair, S. Pons, N. Lin, A. P. Seitsonen, H. Brune, J. V. Barth, and K. Kern, Nat. Mater. 8, 189 (2009).

${ }^{13}$ S. I. Csiszar, M. W. Haverkort, Z. Hu, A. Tanaka, H. H. Hsieh, H.-J. Lin, C. T. Chen, T. Hibma, and L. H. Tjeng, Phys. Rev. Lett. 95, 187205 (2005).

${ }^{14}$ A. Scheybal, T. Ramsvik, R. Bertschinger, M. Putero, F. Nolting, and T. A. Jung, Chem. Phys. Lett. 411, 214 (2005).

${ }^{15}$ H. Wende, M. Bernien, J. Luo, C. Sorg, N. Ponpandian, J. Kurde, J. Miguel, M. Piantek, X. Xu, Ph. Eckhold, W. Kuch, K. Baberschke, P. M. Panchmatia, B. Sanyal, P. M. Oppeneer, and O. Eriksson, Nat. Mater. 6, 516 (2007).

${ }^{16}$ C. F. Hermanns, K. Tarafder, M. Bernien, A. Krüger, Y.-M. Chang, P. M. Oppeneer, and W. Kuch, Adv. Mater. 25, 3473 (2013).

${ }^{17}$ C. Wäckerlin, D. Chylarecka, A. Kleibert, K. Müller, C. Iacovita, F. Nolting, T. A. Jung, and N. Ballav, Nat. Commun. 1, 61 (2010).

${ }^{18}$ J. Miguel, C. F. Hermanns, M. Bernien, A. Krüger, and W. Kuch, J. Phys. Chem. Lett. 2, 1455 (2011).

${ }^{19}$ C. F. Hermanns, M. Bernien, A. Krüger, J. Miguel, and W. Kuch, J. Phys.: Condens. Matter. 24, 394008 (2012).

${ }^{20}$ C. Wäckerlin, K. Tarafder, D. Siewert, J. Girovsky, T. Hählen, C. Iacovita, A. Kleibert, F. Nolting, T. A. Jung, P. M. Oppeneer, and N. Ballav, Chem. Sci. 3, 3154 (2012). 
${ }^{21}$ C. Wäckerlin, J. Nowakowski, S.-X. Liu, M. Jaggi, D. Siewert, J. Girovsky, A. Shchyrba, T. Hählen, A. Kleibert, P. M. Oppeneer, F. Nolting, S. Decurtins, T. A. Jung, and N. Ballav, Adv. Mater. 25, 2404 (2013).

${ }^{22}$ C. Wäckerlin, K. Tarafder, J. Girovsky, J. Nowakowski, T. Hählen, A. Shchyrba, D. Siewert, A. Kleibert, F. Nolting, P. M. Oppeneer, T. A. Jung, and N. Ballav, Angew. Chem. Int. Ed. 52, 4568 (2013).

${ }^{23}$ A. Varykhalov, J. Sánchez-Barriga, A. M. Shikin, C. Biswas, E. Vescovo, A. Rybkin, D. Marchenko, and O. Rader, Phys. Rev. Lett. 101, 157601 (2008).

${ }^{24}$ Yu. S. Dedkov, M. Fonin, U. Rüdiger, and C. Laubschat, Phys. Rev. Lett. 100, 107602 (2008).

${ }^{25}$ See Supplemental Material at http://link.aps.org/supplemental/ 10.1103/PhysRevB.88.104420 for XA and XMCD spectra of the Ni substrate at different fields.

${ }^{26}$ J. Wang, Y. Shi, J. Cao, and R. Wu, Appl. Phys. Lett. 94, 122501 (2009).

${ }^{27}$ A. Palii, B. Tsukerblat, S. Klokishner, K. R. Dunbar, J. M. Clements-Juan, and E. Coronado, Chem. Soc. Rev. 40, 3130 (2011).

${ }^{28}$ J. Klanke, E. Rentschler, K. Medjanik, D. Kutnyakhov, G. Schönhense, S. Krasnikov, I. V. Shvets, S. Schuppler, P. Nagel, M. Merz, and H. J. Elmers, Phys. Rev. Lett. 110, 137202 (2013).
${ }^{29}$ A. Lodi Rizzini, C. Krull, T. Balashov, J. J. Kavich, A. Mugarza, P. S. Miedema, P. K. Thakur, V. Sessi, S. Klyatskaya, M. Ruben, S. Stepanow, and P. Gambardella, Phys. Rev. Lett. 107, 177205 (2011).

${ }^{30}$ S. Stepanow, P. S. Miedema, A. Mugarza, G. Ceballos, P. Moras, J. C. Cezar, C. Carbone, F. M. F. de Groot, and P. Gambardella, Phys. Rev. B 83, 220401 (2011).

${ }^{31}$ B. T. Thole, P. Carra, F. Sette, and G. van der Laan, Phys. Rev. Lett. 68, 1943 (1992).

${ }^{32}$ P. Carra, B. T. Thole, M. Altarelli, and X. Wang, Phys. Rev. Lett. 70, 694 (1993).

${ }^{33}$ J. Stöhr and H. König, Phys. Rev. Lett. 75, 3748 (1995).

${ }^{34}$ H. Bethe, Ann. Phys. (NY) 395, 133 (1929).

${ }^{35}$ G. van der Laan, Physica B 158, 395 (1989).

${ }^{36}$ G. van der Laan and B. T. J. Thole, J. Electron Spectrosc. Relat. Phenom. 86, 57 (1997).

${ }^{37}$ G. van der Laan and B. T. J. Thole, Phys. Rev. B 42, 6670 (1990).

${ }^{38}$ M. Bernien, J. Miguel, C. Weis, Md. E. Ali, J. Kurde, B. Krumme, P. M. Panchmatia, B. Sanyal, M. Piantek, P. Srivastava, K. Baberschke, P. M. Oppeneer, O. Eriksson, W. Kuch, and H. Wende, Phys. Rev. Lett. 102, 047202 (2009).

${ }^{39}$ Y. Li and K. Baberschke, Phys. Rev. Lett. 68, 1208 (1992).

${ }^{40}$ M.-S. Liao, J. D. Watts, and M.-J. Huang, J. Phys. Chem. A 109, 11996 (2005). 